International Journal of Pure and Applied Mathematics

Volume 84 No. 1 2013, 185-192

ISSN: 1311-8080 (printed version); ISSN: 1314-3395 (on-line version)

url: http://www.ijpam.eu

doi: http://dx.doi.org/10.12732/ijpam.v84i1.14

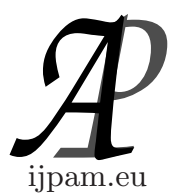

\title{
A NOTE ON *LOCAL COHEN-MACAULAY RINGS
}

\author{
Monica La Barbiera \\ Department of Mathematics \\ University of Messina \\ Viale Ferdinando Stagno d'Alcontres, 31 \\ 98166, Messina, ITALY
}

\begin{abstract}
Some properties of local rings and Cohen-Macaulay modules are investigated in the graduate case.
\end{abstract}

AMS Subject Classification: 13A02, 13A15,13C15,13H10

Key Words: graded rings, local rings, Cohen-Macaulay rings

\section{Introduction}

In this paper we are interested to investigate the Cohen-Macaulay property on graded rings. The notion of Cohen-Macaulay ring is a workhorse of commutative algebra. This notion is sufficiently general to allow a wealth of examples in algebraic geometry, invariant theory and combinatorics ([1], [2]).

Let $R$ be a commutative noetherian ring with unit. Some properties of Cohen-Macaulay $R$-modules and rings are studied ([1]). If $R$ is a graded ring, similar properties can be established and studied. Definitions and results are inspired by the results known in the not graded case, however it is interesting to establish them in the graduate case. In fact because of their importance for algebraic geometry, graded rings have been a standard topic in commutative algebra and for such rings good properties can be determined, as in the local case. In particular characterizations of grades rings are given only use localizations with respect to graded prime ideals. It follows the utility ([1], [3], [4], [5],

Received: January 24, 2013

(c) 2013 Academic Publications, Ltd. url: www.acadpubl.eu 
[6]). The aim of this paper is to investigate some properties of local rings and Cohen-Macaulay $R$-modules in the graduate case.

The paper is organized as follows. In Section 1 some preliminary notions and some properties on graded rings are introduced. Let $R$ be a graded ring and $I$ be an arbitrary ideal of $R . I^{*}$ is the graded ideal generated by all homogeneous elements of $I$ and it is the largest graded ideal contained in $I$. In particular, for each prime ideal $\wp \subset R$, the graded ideal $\wp^{*}$ is a prime ideal too. In [1] there are theorems that link theoretic properties of $\wp$ and $\wp^{*}$ by localizations of finite graded $R$-modules, that is $\operatorname{dim}\left(M_{\wp}\right)=\operatorname{dim}\left(M_{\wp^{*}}\right)+1$ and $\operatorname{depth}\left(M_{\wp}\right)=\operatorname{depth}\left(M_{\wp^{*}}\right)+1$, for all non-graded prime ideal $\wp$ of $R$. We consider the graded counterparts of local rings, namely the *local rings. A graded ideal $m$ of a graded ring $R$ is called *maximal if every graded ideal that properly contains $m$ equals $R$. Then $R$ is a *local ring if it has a unique *maximal ideal. In Section 2 we study an important class of *local rings, namely the *local Cohen-Macaulay rings. A *local noetherian ring $(R, m)$ is a ${ }^{*}$ CohenMacaulay ring if ${ }^{*} \operatorname{dim}(R)={ }^{*} \operatorname{depth}(R)$, where the ${ }^{*} \operatorname{dimension}$ of $R,{ }^{*} \operatorname{dim}(R)$, is the supremum of all numbers $h$ for which there exists a chain of graded prime ideals and the ${ }^{*} \operatorname{depth}(R)$ is the common length of the maximal homogeneous regular sequences in $m$. It is studied the behavior of the ${ }^{*}$ Cohen-Macaulay property under some changes of rings proving in which cases the properties are preserved after a reformulation of statements given in [1]. More precisely, we prove that such property is stable under homogeneous localization and we give a characterization of ${ }^{*}$ Cohen-Macaulay rings.

\section{Preliminary and Notations}

Let $R$ be a commutative noetherian graded ring. In [1] there are some definitions related to the graded ideals of $R$ and some properties on the dimension and the depth of some localizations of finite graded $R$-modules are stated. We recall some of them.

Definition 2.1. Let $I \subset R$ be an ideal (not necessarily graded). $I^{*}$ is the graded ideal of $R$ generated by all homogeneous elements of $I$ (the homogeneous elements of $I$ of degree $j$ lie in $I_{j}=I \cap R_{j}$ ).

The ideal $I^{*}$ is the largest graded ideal contained in $I$. If $I$ is a graded ideal, then $I=I^{*}$.

Example 2.2. Let $I=\left(X_{1}^{2}-2, X_{1} X_{2}-2\right) \subset R=K\left[X_{1}, X_{2}\right]$. We have:

$$
I_{0}=I \cap R_{0}=(0)
$$




$$
\begin{gathered}
I_{1}=I \cap R_{1}=(0) \\
I_{2}=I \cap R_{2}=\left\langle X_{1}^{2}-X_{1} X_{2}\right\rangle,
\end{gathered}
$$

in fact $f=X_{1}^{2}-2-\left(X_{1} X_{2}-2\right)=X_{1}^{2}-X_{1} X_{2} \in I_{2}$. In general, for $i>2$ :

$$
I_{i}=I \cap R_{i}=\left\langle g\left(X_{1}^{2}-X_{1} X_{2}\right)\right\rangle,
$$

with $\operatorname{deg}(g)=i-2$. It follows that $I^{*}=\left(X_{1}^{2}-X_{1} X_{2}\right) \subset I$.

Let $\wp \subset R$ be a prime ideal. In [1] the following property is given.

Lemma 2.3. ([1], 1.5.6) Let $R$ be a graded ring.

1. a) For every prime ideal $\wp$, the ideal $\wp^{*}$ is a graded prime ideal.

2. b) Let $M$ be a graded $R$-module. If $\wp \in \operatorname{Supp}(M)$, then $\wp^{*} \in \operatorname{Supp}(M)$.

We recall the following theorems that link theoretic properties of $\wp$ and $\wp^{*}$ by localizations of finite graded $R$-modules.

Theorem 2.4. ([1], 1.5.8) Let $R$ be a noetherian graded ring, $M$ be a finite graded $R$-module and $\wp \in \operatorname{Supp}(M)$.

1) If $\wp$ is graded, then there exists a chain of graded prime ideals $\wp_{i} \in$ $\operatorname{Supp}(M)$

$$
\wp_{0} \subset \ldots \subset \wp_{d}=\wp, \quad d=\operatorname{dim}\left(M_{\wp}\right) .
$$

2) If $\wp$ is not graded, then $\operatorname{dim}\left(M_{\wp}\right)=\operatorname{dim}\left(M_{\wp^{*}}\right)+1$.

Theorem 2.5. ([1], 1.5.9) Let $R$ be a noetherian graded ring, $M$ be a finite graded $R$-module and $\wp \in \operatorname{Supp}(M)$ be a non-graded prime ideal. Then: $\operatorname{depth}\left(M_{\wp}\right)=\operatorname{depth}\left(M_{\wp^{*}}\right)+1$.

Corollary 2.6. Let $R$ be a noetherian local graded ring and $\wp$ be a non-graded prime ideal. Then:

1) $R_{\wp^{*}}$ is Cohen-Macaulay $\Leftrightarrow R_{\wp}$ is Cohen-Macaulay.

2) $R_{\wp}$ is regular $\Leftrightarrow R_{\wp}$ is regular.

\section{Proof.}

1. 1) If $R_{\wp^{*}}$ is Cohen-Macaulay, then $\operatorname{dim}\left(R_{\wp^{*}}\right)=\operatorname{depth}\left(R_{\wp^{*}}\right)$. One has by Theorems 2.4 and 2.5 that $\operatorname{dim}\left(R_{\wp^{*}}\right)=\operatorname{dim}\left(R_{\wp}\right)-1$ e $\operatorname{depth}\left(R_{\wp^{*}}\right)=$ $\operatorname{depth}\left(R_{\wp}\right)-1$. Hence $\operatorname{dim} R_{\wp}=\operatorname{depth} R_{\wp}$, i.e. $R_{\wp}$ is Cohen-Macaulay. The converse is clearly. 
2. 2) Let $R_{\wp^{*}}$ be a graded regular ring, i.e. its maximal ideal $\wp^{*} R_{\wp^{*}}$ is generated by $n$ homogeneous elements with $\operatorname{dim}\left(R_{\wp^{*}}\right)=n$. Hence ${ }^{*} \wp$ is generated by $n$ elements.

It follows $\operatorname{dim}\left(R_{\wp}\right)=\operatorname{dim}\left(R_{\wp^{*}}\right)+1=n+1$. Being $\wp=\wp^{*}+a R$ with $a \in R \backslash \wp^{*}([1])$, then $\wp$ is generated by $n+1$ elements and $\wp R_{\wp}$ is generated by $n+1$ elements where $\operatorname{dim}\left(R_{\wp}\right)=n+1$, i.e. $R_{\wp}$ is a regular ring. The converse is clearly.

We are interested to a special class of graded rings, namely the *local rings.

Definition 2.7. Let $R$ be a graded ring. A graded ideal $m$ of $R$ is called *maximal if every graded ideal that properly contains $m$ equals $R$.

Definition 2.8. A graded ring $R$ is a *local ring if it has a unique ${ }^{*}$ maximal ideal.

Example 2.9. Let $R$ be a graded ring and $\wp$ be a prime graded ideal of $R$. Let $S$ be the set of the homogeneous elements of $R$ that don't lie in $\wp$. $S$ is a multiplicatively closed set. Denote $R_{(\wp)}$ the homogeneous localization of $R$. $R_{(\wp)}$ is a graded ring and the graduation is defined setting $\left(R_{(\wp)}\right)_{i}=\left\{\frac{x}{a} \in\right.$ $\left.R_{(\wp)} \mid \operatorname{deg}\left(\frac{x}{a}\right)=i\right\}$, where $\operatorname{deg}\left(\frac{x}{a}\right)=\operatorname{deg}(x)-\operatorname{deg}(a)$, with $x$ and $a$ homogeneous elements. Then $R_{(\wp)}$ is a *local ring with *maximal ideal $\wp^{*} R_{(\wp)}$.

Remark 2.10. If $\wp$ is a non-graded ideal of $R$, then the homogeneous localization $R_{(\wp)}$ is a *local ring with ${ }^{*}$ maximal ideal $\wp^{*} R_{(\wp)}=\left\{\frac{p}{s} \mid p \in \wp^{*}, s \in\right.$ $S$, where $S$ is the set of the homogeneous elements of $R$ that don't lie in $\wp$. Note that $\wp^{*} R_{(\wp)}$ is ${ }^{*}$ maximal but not maximal. In fact in $R_{(\wp)}$ there exists the non-graded ideal $\wp R_{(\wp)}$ that contains $\wp^{*} R_{(\wp)}$.

Example 2.11. Let $R=K[X]$ be a graded ring with the standard graduation and $\wp=(X)$ be a graded prime ideal of $R$. One has $S=R_{0} \backslash\{0\}=K \backslash\{0\}$.

$R_{S}=R_{(\wp)}=\left\{\frac{f}{h} \mid f \in R, h \in K \backslash\{0\}\right\}$ is the homogeneous localization of $R$ with respect to $S . R_{(\wp)}$ is a graded ring with the graduation: $\left(R_{(\wp)}\right)_{i}=\left\{\frac{g}{h} \in\right.$ $R_{(\wp)} \mid g \in R, h \in K \backslash\{0\}$ homogeneous with $\left.\operatorname{deg}(g)-\operatorname{deg}(h)=i\right\}=\left\{\frac{c X^{i}}{h} \in\right.$ $\left.R_{(\wp)} \mid c X^{i} \in R_{i}, h \in K \backslash\{0\}\right\} \cong R_{i}$. Then $R_{(\wp)}$ is a *local ring with *maximal ideal $\wp R_{(\wp)}=\left\{\frac{p}{s} \mid p \in \wp, s \in S\right\}=\left\{\frac{f X}{s} \mid f \in R, s \in K \backslash\{0\}\right\}$.

Example 2.12. Let $R=K[X]$ be a graded ring with the standard graduation and $\wp=(X+1)$ be a non-graded prime ideal of $R$ with $\wp^{*}=$ (0). $S$ is the set of the homogeneous elements of $R$ defined as follows $S=$ $\left\{c_{0}, c_{1} X, c_{2} X^{2}, c_{3} X^{3}, \ldots, c_{i} X^{i}, \ldots\right\} \backslash\{0\}, c_{i} \in K$, with $c_{i} X^{i} \notin \wp$.

$R_{S}=R_{(\wp)}=\left\{\frac{f}{c_{i} X^{i}} \mid f \in R, c_{i} X^{i} \in S\right\}$ is the homogeneous localization of $R$ with respect to $S . R_{(\wp)}$ is a graded ring with graduation: $\left(R_{(\wp)}\right)_{j}=\left\{\frac{f}{c_{i} X^{i}} \in\right.$ 
$R_{(\wp)} \mid f, c_{i} X^{i}$ homogeneous in $R$ with $\left.\operatorname{deg}(f)-\operatorname{deg}\left(c_{i} X^{i}\right)=j\right\}$. Then $R_{(\wp)}$ is a *local ring with *maximal ideal $\wp^{*} R_{(\wp)}=\left\{\frac{p}{s} \mid p \in \wp^{*}, s \in S\right\}=\left\{\frac{0}{s} \mid s \in S\right\}$ being $\wp^{*}=(0)$. One has $\wp^{*} R_{(\wp)} \subseteq \wp R_{(\wp)}$, with $\wp R_{(\wp)}=\left\{\frac{h}{s} \mid h \in \wp, s \in S\right\}$ non-graded ideal of $R_{(\wp)}$. Hence $\wp^{*} R_{(\wp)}$ is a ${ }^{*}$ maximal ideal but not maximal.

\section{3. * Local Cohen-Macaulay Rings}

In this section we introduce an important class of *local rings, namely the *local Cohen-Macaulay rings. We give the following definitions.

Definition 3.1. Let $R$ be a *local ring. Let $\wp$ be a graded prime ideal of $R$. The *height of $\wp$ is the supremum of the lengths of all chains of graded prime ideals $\wp_{0} \subset \wp_{1} \subset \cdots \subset \wp_{h}=\wp$. Let denote it ${ }^{*} h t(\wp)$.

Definition 3.2. Let $R$ be a *local ring. Let $I$ be a graded ideal of $R$. The ${ }^{*}$ height of $I$, denoted ${ }^{*} h t(I)$, is defined as ${ }^{*} \operatorname{ht}(I)=\min \left\{{ }^{*} h t(\wp) \mid I \subset\right.$ $\wp, \wp$ graded prime ideal\}.

Definition 3.3. Let $R$ be a ${ }^{*}$ local ring with *maximal ideal $m$. The *dimension of $R$ is the supremum of all numbers $h$ for which there exists a chain of graded prime ideals $\wp_{0} \subset \wp_{1} \subset \cdots \subset \wp_{h}$ in $R$. Let denote it ${ }^{*} \operatorname{dim}(R)$.

\section{Remark 3.4.}

1) Let $R$ be a ${ }^{*}$ local ring with ${ }^{*}$ maximal ideal $m$. Then ${ }^{*} \operatorname{dim}(R)={ }^{*}$ ht $(m)$.

2) Let $R_{\wp}$ be a *local ring with $\wp$ graded ideal, then ${ }^{*} \operatorname{dim}\left(R_{\wp}\right)={ }^{*} h t(\wp)$.

Definition 3.5. A sequence $x_{1}, \ldots, x_{n}$ of homogeneous elements of a graded ring $R$ is called *regular sequence on $R$ (or homogeneous $R$-sequence) if the following conditions are satisfied:

1) $\left(x_{1}, \ldots, x_{n}\right)$ is a proper graded ideal;

2) $x_{i+1}$ is a regular homogeneous element in $R /\left(x_{1}, \ldots, x_{i}\right)$, for $i=0, \ldots, n-$ 1.

Definition 3.6. Let $R$ be a graded ring, $M$ be a finite graded $R$-module. A sequence $\underline{x}=\left\{x_{1}, \ldots, x_{n}\right\}$ of homogeneous elements of $R$ is called $M-{ }^{*}$ regular sequence (or homogeneous $M$-sequence) if the following conditions are satisfied: 1) $\underline{x} M \neq M$;

2) $x_{i}$ is a nonzero-divisor in $M /\left(x_{1}, \ldots, x_{i-1}\right) M$, for $i=1, \ldots, n$.

Definition 3.7. Let $R$ be a graded ring, $M$ be a finite graded $R$-module and $I$ be an ideal of $R$ such that $I M \neq M$. The common length of the maximal 
homogeneous $M$-sequences in $I^{*}$ is called the ${ }^{*}$ grade of $I$ on $M$, denoted by ${ }^{*} \operatorname{grade}(I, M)$.

A special situation occurs for *local rings:

Definition 3.8. Let $(R, m)$ be a *local noetherian ring and $M$ be a finite graded $R$-module. The ${ }^{*}$ grade of $m$ on $M$ is called the ${ }^{*}$ depth of $M$, denoted by ${ }^{*} \operatorname{depth}(M)$.

Let $I$ be an ideal of a graded ring $R$. It is customary to set: ${ }^{*}$ grade $(I)=$ ${ }^{*} \operatorname{grade}(R / I)={ }^{*} \operatorname{grade}(I, R)$. More precisely we give the following:

Definition 3.9. Let $I$ be an ideal of $R$. The ${ }^{*}$ grade of $I$ is the common length of the maximal homogeneous $R$-sequences in $I^{*}$, denoted ${ }^{*}$ grade $(I)$.

Definition 3.10. A *local noetherian ring $(R, m)$ is a ${ }^{*}$ Cohen-Macaulay ring if $* \operatorname{dim}(R)={ }^{*} \operatorname{depth}(R)$.

Remark 3.11. Let $R$ be an arbitrary noetherian graded ring. $R$ is a ${ }^{*}$ Cohen-Macaulay ring if $R_{m}$ is a ${ }^{*}$ Cohen-Macaulay ring for all *maximal ideals of $R$.

Example 3.12. Let $R=K[X, Y] /(X Y) \cong K[x, y]$ with $x y=0$.

The ideal $m=(x, y)$ is the only *maximal ideal of $R$, hence $R$ is a *local ring.

$R$ is ${ }^{*}$ Cohen-Macaulay if ${ }^{*} \operatorname{depth}(R)={ }^{*} \operatorname{dim}(R)$.

One has ${ }^{*} \operatorname{dim}(R)=1$. In fact ${ }^{*} \operatorname{dim}(R)={ }^{*} h t(m)=1$ because the chains of graded prime ideals contained in $m$ are $(x) \subset(x, y)$ and $(y) \subset(x, y)$.

One has ${ }^{*} \operatorname{depth}(R)=1$. In fact, in general ${ }^{*} \operatorname{depth}(R) \leq{ }^{*} \operatorname{dim}(R)$, hence ${ }^{*} \operatorname{depth}(R) \leq 1$ and ${ }^{*} \operatorname{depth}(R)={ }^{*} \operatorname{depth}(m)$. Note that $x \in m$ and $y \in m$ are not regular elements in $R$ (being $x y=0$ ). But $x+y \in m$ is a homogeneous regular element in $m$. It follows ${ }^{*} \operatorname{depth}(R)={ }^{*} \operatorname{depth}(m)=1$.

Definition 3.13. Let $R$ be a noetherian *local ring. A finite graded $R$-module $M$ is ${ }^{*}$ Cohen-Macaulay if ${ }^{*} \operatorname{dim}(M)={ }^{*} \operatorname{depth}(M)$.

A *maximal Cohen-Macaulay module is a ${ }^{*}$ Cohen-Macaulay module $M$ such that ${ }^{*} \operatorname{dim}(R)={ }^{*} \operatorname{dim}(M)$.

Remark 3.14. Let $R$ be an arbitrary noetherian graded ring. $M$ is a ${ }^{*}$ Cohen-Macaulay module if $M_{m}$ is a ${ }^{*}$ Cohen-Macaulay module for all *maximal ideals $m \in \operatorname{Supp}(M)$.

We give some properties of ${ }^{*}$ Cohen-Macaulay modules. The following result states that the ${ }^{*}$ Cohen-Macaulay property is stable under homogeneous localization. 
Theorem 3.15. Let $R$ be a noetherian *local ring, $M$ be a finite ${ }^{*}$ CohenMacaulay module. The localized graded module $M_{(\wp)}$ is ${ }^{*}$ Cohen-Macaulay for all graded prime ideal $\wp \in \operatorname{Spec}(R)$.

Proof. Assume $M_{(\wp)} \neq 0$. We use induction on ${ }^{*} \operatorname{depth}\left(M_{(\wp)}\right)$.

If $* \operatorname{depth}\left(M_{(\wp)}\right)=0$, then $\wp \in \operatorname{Ass}(M)$ and $\wp$ is a minimal graded prime of $\operatorname{Supp}(M)([1])$. Therefore ${ }^{*} \operatorname{depth}\left(M_{(\wp)}\right)=0$. The same argument shows that $\wp$ can not be contained in any graded ideal $Q \in \operatorname{Ass}(M)$ if ${ }^{*} \operatorname{depth}\left(M_{(\wp)}\right)>0$. So $\wp$ contains a homogeneous $M$-regular element $x$, and the induction hypothesis applies to $M / x M$. It follows easily that $M_{(\wp)}$ is ${ }^{*}$ local Cohen-Macaulay.

Theorem 3.16. A noetherian graded ring $R$ is ${ }^{*}$ Cohen-Macaulay if and only if for every homogeneous ideal $I$ generated by $n$ elements, where $n=$ ${ }^{*} h t(I)$, the associated graded prime ideals of $R / I$ are the minimal prime ideals of $I$.

Proof. $\Rightarrow)$ Suppose $I=\left(x_{1}, \ldots, x_{n}\right)$, where $x_{1}, \ldots, x_{n}$ are homogeneous elements of $R$. Let $\wp_{1}, \wp_{2} \in \operatorname{Ass}(R / I)$ be graded ideals and $\wp_{1} \subset \wp_{2}$. Then there is a ${ }^{*}$ maximal ideal $m$ with $\wp_{1} \subset \wp_{2} \subset m$ and $\wp_{1} R_{m}, \wp_{2} R_{m} \in \operatorname{Ass}\left(R_{m} / I_{m}\right)$. If ${ }^{*}$ ht $(I)=n$, then ${ }^{*} \operatorname{dim}\left(R_{m} / I_{m}\right)={ }^{*} \operatorname{dim}\left(R_{m}\right)-n$ and $x_{1}, \ldots, x_{n}$ is a homogeneous $R_{m}$-sequence ([1]). Therefore $R_{m} / I_{m}$ is ${ }^{*}$ Cohen-Macaulay, hence $\wp_{1} R_{m}=\wp_{2} R_{m}$ and $\wp_{1}=\wp_{2}$.

$\Leftarrow)$ Let $J \subset R$ be a graded ideal and suppose ${ }^{*} h t(J)=n$. Then there exists a sequence of homogeneous elements $x_{1}, \ldots, x_{n} \in J$ with ${ }^{*} h t\left(x_{1}, \ldots, x_{i}\right)=i$ for all $i=1, \ldots, n$. It is not possible for $x_{i+1}$ to be contained in a minimal graded ideal $\left(x_{1}, \ldots, x_{i}\right)$. By hypothesis it is a homogeneous $R /\left(x_{1}, \ldots, x_{i}\right)$ regular element. So $x_{1}, \ldots, x_{n}$ is a homogeneous $R$-sequence. This proves that ${ }^{*} \operatorname{ht}(J)={ }^{*} \operatorname{grade}(J)$ for every graded ideal $J$ of $R$. Hence $R$ is *Cohen-Macaulay.

\section{References}

[1] W. Bruns, J. Herzog, Cohen-Macaulay Rings, Cambridge Studies in Advanced Mathematics (1998).

[2] D. Eisenbud, Commutative Algebra with a View Toward Algebraic Geometry, Springer (1995).

[3] M. La Barbiera, Conditions $S_{q}$ and $G_{q}$ on graded rings, Atti dell'Accademia Peloritana dei Pericolanti, LXXXIV, C1A0601007 (2006).

[4] M. La Barbiera, On graded ${ }^{*} G_{q}$-rings, International Mathematical Forum, 5 (2010), 191-198. 
[5] M. La Barbiera, A note on * complete intersections generated by linear forms, Preprint.

[6] M. La Barbiera, On the symmetric algebra of graded modules and torsion freeness, Preprint. 